\title{
PRELIMINARY RESULTS ON INTERMITTENT BEHAVIOUR OF MILLISECOND PULSAR SAX J1808.4-3658
}

\author{
M. T. Menna, ${ }^{1}$ A. Papitto, ${ }^{1}$ L. Burderi, ${ }^{1}$ T. Di Salvo,${ }^{2}$ L. Stella, ${ }^{1}$ and N. R. Robba ${ }^{3}$
}

We analyzed RXTE data from the burst of the year 2000 of the X-ray millisecond binary pulsar SAX J1808.4-3658 with the intent of determining the new orbital parameters.

We used the observations of SAX J1808.4-3658 performed by the Rossi X-Ray Timing Explorer (RXTE) when the source was again detected in outburst during the period Jinnuary-March 2000 (Wijnands et al. 2001). In particular we examined the data from the Proportional Counter Array (PCA) (Jahoda et al. 1996). We first applied barycentric correction to the data using the optical coordinates of the source (Roche et al. 98).

This burst is much weaker than the previous 1998 burst. We extracted a series of light curves to determine intensity levels and have therefore focused our attention on the obscrvation of the 2nd of Feb 2000, wich has a count rate, background subtracted, consistent with a constant value of about $143 \mathrm{cts} / \mathrm{s}$, one of the highest levels, where pulsations are most likely to be detected. Having verified the presence of pulsing behaviour calculating a power spectrum density which clearly shows the harmonic line at about 401 $\mathrm{Hz}$, we then proceed to perfom a series of epoch folding searches at the nominal spin period to follow the spin evolution along time and determine new orbital parameters.

The epoch folding scarches are to be perfomed folding a number of spin cycles long enough to have enough photons to detect a signal, but short enough so that the spin period is not affected by the orbital motion. We fold over intervals of $100 \mathrm{~s}$. We report in Fig. 1 an example of one of the subsets we found of Time (in seconds) us Spin Period (in units of $\left.10^{-3} \mathrm{~s}\right)$. We then performed a global fit along all the time span of the observation (16 days) with a constant plus sinus function of the kind $f(x)=c+A \sin (\nu t+\phi)$ and obtained the following values for the phisical quantities: spin period

\footnotetext{
${ }^{1}$ Osservatorio Astronomico di Roma, Monteporzio Catone (Roma), via di Frascati 33, 00040, Italy (menna@mporzio. astro.it).

${ }^{2}$ Astronomical Institute Cniversity of Amsterdam.

${ }^{2}$ Dipartimento di Astronomia, Cniversità di Palermo.
}

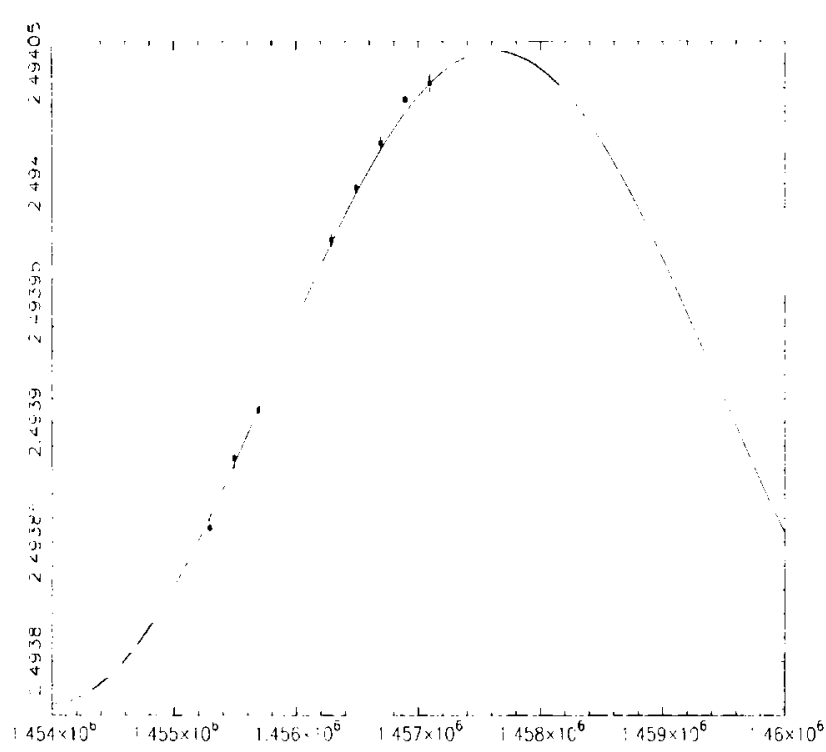

Fig. 1. Example plot of Time vs Spin Periods. The continuous curve represents the best fit.

$P_{\text {spin }}=2.49391832810^{-3} s+/-110^{-9}$, orbital pe$\operatorname{riod} P_{\text {orb }}=7248.95 \mathrm{~s}+/-0.09 \mathrm{~s}$, epoch of $\pi / 2$ mean longitude $T_{\text {nod }}=51576.1112 \mathrm{MJD}+/-210^{-4}$.

The puzzling behaviour that emerged is that, notwithstanding the constant flux level, on this time scale pulsations are not present in all the intervals. It can be seen in Fig. 1 by the lack of points along some zones of the curve which has been thoroughly explored. We are currently investigating in more detail the presence of this intermittent pulsating behaviour in the 2000 outburst data (Menna et al. 2004, in preparation).

\section{REFERENCES}

Roche, P. et al. 1998, IAUC, 6885

Jahoda, K. et al. 1996, Proc. SPIE, 2808, 59

Wijnands, R. et al. 2001, ApJ, 560, 892

Menna, M.T. et al. 2004, in preparation 